\title{
Antagonism of Angiotensin II AT1 Receptor and Silencing of CD44 Gene Expression Inhibit Cardiac Fibroblast Activation via Modulating TGF- $\beta 1 /$ Smad Signaling Pathway
}

\author{
Feng Bai ${ }^{1}$, Guangzhao Yang ${ }^{1}$, Joshua Robert Eskew ${ }^{2}$, Ningping Wang ${ }^{2}$, Himangshu Bose ${ }^{2}$, \\ Zhiqing Zhao ${ }^{1,2^{*}}$ \\ ${ }^{1}$ Department of Cardiology, The First Affiliated Hospital of Shanxi Medical University, Taiyuan, China \\ ${ }^{2}$ Cardiovascular Research Laboratory, School of Medicine, Mercer University, Savannah, GA, USA \\ Email: ^zhao_z@mercer.edu
}

How to cite this paper: Bai, F., Yang, G.Z., Eskew, J.R., Wang, N.P., Bose, H. and Zhao, Z.Q. (2020) Antagonism of Angiotensin II AT1 Receptor and Silencing of CD44 Gene Expression Inhibit Cardiac Fibroblast Activation via Modulating TGF- $\beta 1 /$ Smad Signaling Pathway. Advances in Bioscience and Biotechnology, 11, 123-139.

https://doi.org/10.4236/abb.2020.114010

Received: March 9, 2020

Accepted: April 19, 2020

Published: April 22, 2020

Copyright $\odot 2020$ by author(s) and Scientific Research Publishing Inc. This work is licensed under the Creative Commons Attribution International License (CC BY 4.0).

http://creativecommons.org/licenses/by/4.0/

\section{(c) (i) Open Access}

\begin{abstract}
Angiotensin II (Ang II) is known to elicit cardiac fibrosis by activating the AT1 receptor and CD44 expression in the in vivo model. However, the cellular/molecular mechanisms underlying cardiac fibrosis are still not well understood. This study examines the roles of the AT1 receptor and CD44 gene expression in collagen synthesis through Ang II stimulated cardiac fibroblasts. Fibroblasts were isolated from the neonatal rat hearts; the activation of fibroblasts was evaluated using the assays of cell viability and migration, and silencing of CD44 gene expression was conducted with small interfering RNA (siRNA). Results showed that Ang II significantly increases the cell proliferation and migration in a dose-dependent manner. Upon activation, the protein levels of TGF- $\beta 1$, Smad2, Smad4 and collagen I were significantly increased (all $\mathrm{p}<0.05$ vs. unstimulated cells), but these changes were significantly downregulated by the AT1 receptor blocker, telmisartan (all p $<0.05$ vs. Ang II activated cells). Furthermore, mRNA and protein level of CD44 were upregulated, and there was a linear correlation between CD44 and TGF- $\beta 1$ as demonstrated by Pearson correlation analysis $(r=0.955, \mathrm{p}<0.01)$. Gene transfection of fibroblasts with Ad-CD44 siRNA, as evidenced by low levels of CD44 mRNA and protein, significantly reduced the production of collagen I. In summary, these results indicate that the proliferation, migration and collagen production from Ang II activated cardiac fibroblasts are potentially mediated by the AT1 receptor and CD44. Such a signaling mechanism could be crucial for the production of collagen and the development of tissue fibrosis in the heart.
\end{abstract}




\section{Keywords}

Angiotensin II AT1 Receptor, CD44, Collagen, Fibroblasts, Telmisartan

\section{Introduction}

Cardiac fibroblasts, defined as cells of the non-cardiomyocytes in the heart, are usually spindle-shaped cells with a large oval/flat pleomorphic nucleus, which can be found in the interstitial spaces of myocardium [1]. In the normal conditions, cardiac fibroblasts provide a structural scaffold for cardiomyocytes by producing fibrillar collagens as the predominant components of extracellular matrix proteins to maintain the structural integrity of the heart [2] [3]. Upon mechanical or chemical injury to the myocardium, the population of cardiac fibroblasts increases through the replication of resident fibroblasts, transformation of endothelial cells or migration of bone marrow cells. Excessive production and deposition of extracellular matrix proteins from cardiac fibroblasts lead to the development of cardiac fibrosis with adverse effects on myocardial structure and function [4] [5] [6].

The CD44 antigen is a transmembrane glycoprotein, which is primarily expressed in a large number of mammalian cell types such as inflammatory and vascular cells, and involved in cellular adhesion and migration [7]. The principal ligand of CD44 is hyaluronan, a major glycosaminoglycan constituent of extracellular matrix that is upregulated within the interstitium of myocardium along with activation of cardiac fibroblasts. Cell surface CD44 on cardiac fibroblasts is an adhesion receptor for hyaluronic acid, and facilitates the proliferation and migration of fibroblasts into fibrin matrices. Activated cardiac fibroblasts, in turn, produce excessive fibrotic extracellular matrix, and cause cardiac fibrosis and cardiomyocyte hypertrophy [8].

In the rat model of angiotensin II (Ang II) infusion, we have previously reported that the blockade of Ang II AT1 receptor with a selective AT1 receptor blocker telmisartan reduces myocardial fibrosis, which is primarily mediated by inhibiting differentiation of fibroblasts to myofibroblasts via downregulating the expression of transforming growth factor- $\beta 1$ (TGF- $\beta 1$ ) released from the infiltrated macrophages. In coincidence with this blockade of the AT1 receptor, CD44 protein expression in the myocardium was downregulated [9]. Furthermore, CD44 deficiency reduced Ang II-induced myocardial fibrosis through inhibiting inflammatory cytokines and oxidative stress [10]. It is well known that fibroblasts play a key role in mediating fibrotic cascade after the fibroblasts are differentiated to myofibroblasts in response to TGF- $\beta 1$ stimulation [9]. However, in the majority of previous observations including our studies, the blockade of the AT1 receptor was examined using homogenized tissue blocks from the whole heart, and the expression of CD44 on the microvessels and myocardium was identified using immunohistochemical staining [9] [10] [11] [12] [13]. Fur- 
thermore, although much research has been performed examining the fibroblast activation by Ang II in various organs, at the present time, we do not know whether Ang II stimulation or upregulation of CD44 gene expression has direct positive effect on activation, proliferation and migration of cardiac fibroblasts. Therefore, using isolated cardiac fibroblasts from the neonatal heart, we examined whether migration/differentiation of cardiac fibroblasts stimulated by Ang II could be blocked by the AT1 receptor blocker, telmisartan, and whether silencing of CD44 gene expression could inhibit the activation of cardiac fibroblasts. Examination of this phenomenon in cultured fibroblasts may provide the experimental evidence to facilitate the exploration of anti-fibrosis therapy in which the inhibition of cardiac fibroblast activation is proposed as a main target.

\section{Materials and Methods}

\subsection{Antibodies}

Primary antibodies against cardiac troponin I (ab47003), Vimentin (ab8978), TGF- $\beta 1$ (ab25121), collagen I (ab90395) were purchased from Abcam (England). Primary antibodies against DDR2 (N-20) (sc7555), von Willebrand Factor (vWF) (sc27649) were purchased from Santa Cruz Biotechnology (Dallas, TX, USA). Primary antibodies against p-Smad2 (No. 3108) and Smad4 (No. 9515) were purchased from Cell Signaling Technology (Boston, MA, USA). Primary antibody against CD44 (bs2507R) was purchased from Bioss Technology (Beijing, China). Donkey anti-Mouse IgG, Alexa Fluor 488 (A21202) and Donkey anti-Goat IgG, Alexa Fluor 594 (A11058) were purchased from Life Technologies (Waltham, MA, USA). Goat anti-Rabbit IgG (CW0103) and Goat anti-Mouse IgG (CW0102) were purchased from ComWin Biotch Co. (Beijing, China). Type II collagenase (17101-015) and angiotensin II (A9525) were purchased from Thermo Fisher Scientific (Waltham, MA, USA) and Sigma-Aldrich (St. Louis, MO, USA) respectively.

\subsection{Isolation and Culture of Neonatal Rat Cardiac Fibroblasts}

All procedures were performed in compliance with "The Guide for the Care of Use of Laboratory Animals" published by the US National Institute of Health (8th edition) and revised in 2011. The experimental protocol was approved by the Experimental Animal Management Committee of Shanxi Medical University, Taiyuan, China (Approval NO. 2015-0001).

One to three-day old Sprague-Dawley rats were obtained from the Animal Laboratory Center of Shanxi Medical University. Cardiac fibroblasts were isolated from rats using a modified version of the differential attachment method as described previously [13]. In brief, the intact hearts were rapidly removed into the cold phosphate buffered saline to wash and rinse out the blood for 3-4 times. After excluding the connective tissue and atrium, the ventricles were minced into 1 cubic $\mathrm{mm}$ tissue blocks and digested with mixed enzyme solution that contains $0.08 \%$ trypsin and $0.04 \%$ type II collagenase through a 6 min-period of 8 - 
10 cycles at $37^{\circ} \mathrm{C}$. The minced tissue solution was filtered through a $70 \mu \mathrm{m}$-cell strainer and centrifuged at $800 \mathrm{~g}$ for $10 \mathrm{~min}$ at room temperature. The cells collected were plated in the wells with moderate DMEM medium supplemented with $10 \% \mathrm{FBS}$, and then incubated at $95 \% \mathrm{O}_{2}$ and $5 \% \mathrm{CO}_{2}$ under $37^{\circ} \mathrm{C}$. Two hours later, unattached cells were discarded and the attached cells were plated in fresh DMEM medium containing $10 \% \mathrm{FBS}$ at $37^{\circ} \mathrm{C}$. When the degree of cell fusion reached to about $80 \%$, the cells were digested with trypsin EDTA and passaged. Subsequent experiments were performed using cells at 2 - 4 passages. Before each assay, isolated cardiac fibroblasts were incubated and synchronized with serum-free DMEM medium for $24 \mathrm{~h}$. In the following observations, the cells were exposed to a fresh medium solution (control), Ang II activation (10 $\mathrm{nM}$ to $1 \mu \mathrm{M})$ or Ang II $(50 \mathrm{nM})$ plus telmisartan $(100 \mathrm{nM})$. The selection of telmisartan dose was based on its inhibition on collagen secretion and production from Ang II stimulated cardiac fibroblasts [14].

\subsection{Immunofluorescence Staining}

Immunofluorescence staining with vimentin and DDR2 (N-20) for fibroblasts, vWF for endothelial cells and cardiac troponin I for cardiomyocytes were selected to identify whether isolated cells are cardiac fibroblasts, but not endothelial cells and cardiomyocytes. In brief, the cells were plated at a density of $1 \times 10^{4}$ per well in 96-well plates, fixed with $4 \%$ paraformaldehyde, and then washed with $0.5 \%$ Triton X-100. Immunofluorescence staining was performed on incubated cells with vimentin (dilution at 1:50), DDR2 (N-20), vWF and cardiac troponin I (all dilution at 1:100), respectively, at $4^{\circ} \mathrm{C}$ overnight. The secondary antibodies including Donkey anti-mouse IgG, Alexa Fluor 488 or Donkey anti-goat IgG, Alexa Fluor 594 were then applied for $90 \mathrm{~min}$ at room temperature correspondingly. The nuclei were stained with DAPI for $10 \mathrm{~min}$. Finally, the morphology of the cells were observed and the photos were taken under the fluorescence microscopy using a digital camera (Olympus, Japan)

\subsection{Cell Proliferation Assay}

Cell proliferation assay was assessed by a Cell Counting Kit-8 (CCK-8, Dojindo, kumamoto, Japan) according to the manufacturer's protocol. Cardiac fibroblasts were seeded into 96-well plates at a density of $7 \times 10^{3}$ cell per well, and treated with Ang II at different concentrations for $24 \mathrm{~h}$. CCK- 8 reagents were then added into the culture medium at a ratio of $1: 10$ at $37^{\circ} \mathrm{C}$ for $2 \mathrm{~h}$ to identify the cell proliferation based on manufacture's instruction (Dojindo, Shanghai, China). The absorbance at $450 \mathrm{~nm}$ was measured using a microplate reader (Molecular Devices Corp, San Jose, CA, USA) to estimate cell proliferation.

\subsection{Transwell Migration Assay}

The transwell migration assay is a commonly used test to study the migratory response of the cells to oxidative stress stimulation. In brief, the cell suspension 
before assay was prepared at the density about $1 \times 10^{6} / \mathrm{ml}$. After adding $500 \mu \mathrm{l}$ serum free medium into 24-well plates, the transwell chambers (No. 3422, CORNING, NY, USA) were placed; $200 \mu$ of cell suspension was pipetted into the upper chamber and cultured at $37^{\circ} \mathrm{C}$ for $12 \mathrm{~h}$ without touching the membrane or introducing air bubbles. Following an incubation period ( $24 \mathrm{~h})$, the migrated cells through membrane were fixed using $4 \%$ paraformaldehyde at room temperature for $30 \mathrm{~min}$. After washing with PBS twice, the membranes were stained by $0.1 \%$ crystal violet solution for $20 \mathrm{~min}$. To count the cell migration, the transwell chamber was viewed using an inverted microscope (Olympus, Japan), and the numbers of migrated cells were counted from eight high-powered fields (HPF, 400× magnification) to get an average sum of the cells.

\subsection{Real-Time Polymerase Chain Reaction (RT-PCR)}

The level of CD44 mRNA detected from the cells was measured by real-time PCR. Briefly, $0.5 \mu \mathrm{g}$ of total RNA isolated from the cells using RNAiso plus (9108, TaKaRa, Shiga, Japan) was reverse-transcribed into cDNA by the Prime Script RT Master Mix (DRR036A, TaKaRa, Shiga, Japan). SYBR Premix Ex TaqTM II (rr820A, TaKaRa, Shiga, Japan) was selected to test CD44 mRNA expression. The thermal profile for SYBR Green PCR was $95^{\circ} \mathrm{C}$ for $30 \mathrm{~s}$, followed by 40 cycles of denaturation at $95^{\circ} \mathrm{C}$ for $5 \mathrm{~s}$ and annealing at $60^{\circ} \mathrm{C}$ for $20 \mathrm{~s}$. The primer sequences were as follows:

CD44 (Gen-Bank accession number, NM_012924.2), forward:

5'-CTCAAGTGGGAATCAAGACAGTGG-3' and reverse:

5'-TGCAGACGGCAAGAATCAGAG-3', GAPDH (Gen-Bank ID NM_017008.3), forward: 5'-GGCACAGTCAAGGCTGAGAATG-3' and reverse:

5'ATGGTGGTGAAGACGCCAGTA-3'. The amplification was normalized to GAPDH and the expression of CD44 mRNA was quantified by the relative quantitative $2^{-\Delta \Delta C t}$ method.

\subsection{Western Blot Analysis}

Isolated cells from different groups were washed with PBS and lysed with lysates buffer (P0013, Beyotime, Shanghai, China) containing protease inhibitors (R0100, Solarbio, Beijing, China) and phosphatase inhibitor (AR1183, Solarbio, Beijing, China) for $30 \mathrm{~min}$ on ice. The supernatant was collected after centrifugation at $14,000 \mathrm{rpm}$ for $15 \mathrm{~min}$ at $4^{\circ} \mathrm{C}$ and the protein concentration was determined using BCA Protein Assay kit (23225, Thermo Fisher Scientific, Waltham, MA, USA). The protein sample $(50 \mu \mathrm{g})$ was loaded, separated using SDS-PAGE, transferred to PVDF membranes at $15 \mathrm{eV}$ for $15 \mathrm{~min}$, and blocked with $5 \%$ non-fat milk for $1 \mathrm{~h}$ at room temperature. The membranes were then incubated with primary antibodies (i.e. CD44, pSmad2, Smad4, TGF- $\beta_{1}$, Collagen I) overnight at $4^{\circ} \mathrm{C}$. After washing 3 times, the membranes were incubated with horseradish peroxidase conjugated secondary antibodies (i.e. goat anti-Rabbit IgG and 
goat anti-Mouse IgG). Finally, the bands were detected with Bio-Rad imaging system assisting with ECL reagent kit according to the manufacturer's instructions. The gray-scale values of straps were analyzed via ImageJ software, and the relative expression of proteins was normalized on $\beta$-actin or GADPH.

\subsection{Adenovirus Infection}

CD44 on the cells was silenced using small interfering RNA (siRNA). The cells in the log phase of growth were separately transfected with adenovirus targeting rat CD44 (Ad-CD44 shRNA1, Ad-CD44 shRNA2 and Ad-CD44 shRNA3) according to the manufacturer's recommendations, which were designed by HANBIO Company (Shanghai, China). A similar adenoviral vector encoding the GFP gene (Ad-GFP NC) was used as a control. After adenovirus were co-incubated with cells for $4 \mathrm{~h}$, the fresh medium was changed and the cells were continually cultured for another $32 \mathrm{~h}$. Target cells showing green fluorescent protein (GFP)-positive were observed under a fluorescence microscope. The virus that induces the most significant decrease in CD44 expression was selected for ongoing experiments. All procedures involving virus operation were performed in a biosafety cabinet.

\subsection{Statistical Analysis}

SPSS 21.0 software served as the statistical analysis tool. Data from six individual experiments were averaged as mean \pm standard deviation and analyzed by one-way ANOVA. Pearson correlation test was used as correlation analysis. A p value of less than 0.05 was considered to be statistically significant.

\section{Results}

\subsection{Identification of Primary Cultured Neonatal Rat Cardiac Fibroblasts}

The first passage of neonatal rat cardiac fibroblasts grew rapidly on day 1 . The cell passaging or splitting of fibroblasts on day 2 displayed their typical spread morphology to form a confluent monolayer culture. Inactive fibroblasts were smaller, spindle or polygonal-shaped, and irregularly branched cytoplasm with a large ovoid nucleus, clear boundaries and good refraction (Figure 1(A)). The purity of isolated cardiac fibroblasts during cell preparations was evaluated using immunofluorescence staining. As shown in Figure 1(B), the majority of the cells were stained positively with vimentin and discoid in domain receptor-2 (DDR2), the markers of fibroblasts. Absence of staining in these cells with cardiac troponin I or von Willebrand Factor indicated that the cells isolated are not cardiomyocytes and endothelial cells. To identify whether cardiac fibroblasts attach to the plates during the culture period, DAPI (4',6-Diamidino-2-Phenylindole, Dihydrochloride), a cell-permeable fluorescent DNA-binding dye, was applied. Intensity of fluorescence staining showed the smaller, more intensely stained nuclei, suggesting that cardiac fibroblasts equally distribute well (Figure 1(B)). 
A
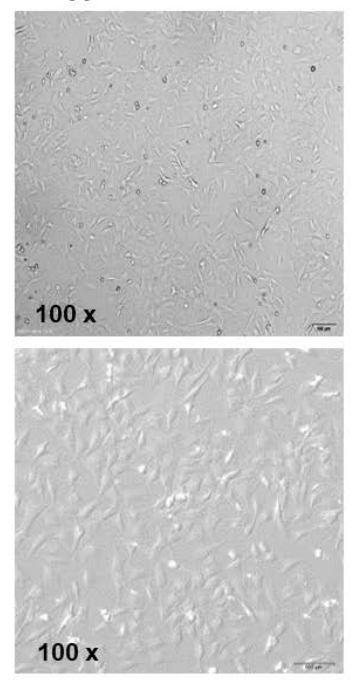

Morphology of fibroblasts
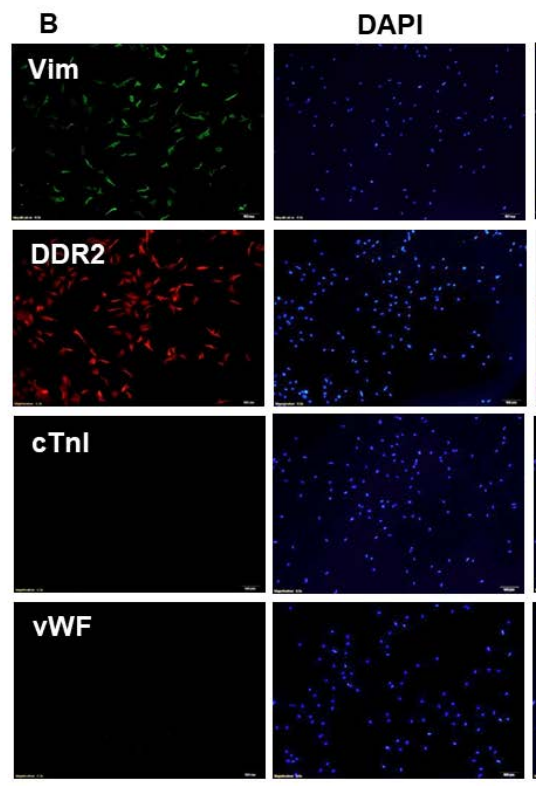

\section{Merged}
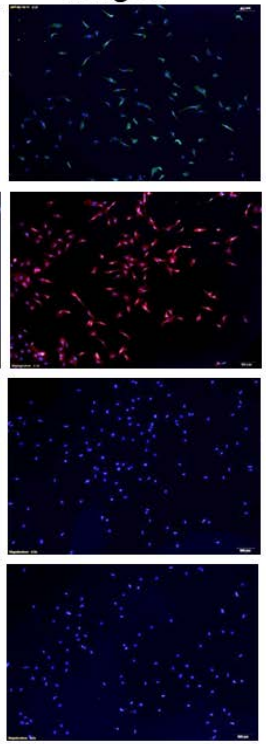

Figure 1. Characterization of cultured cardiac fibroblasts. (A) Cells were isolated from the neonatal rat heart and morphology of second passage of cardiac fibroblasts was identified from the bright-field images taken at $100 \times$ magnification under perspective condition of plane (top panel) and stereoscopic visual condition (bottom panel), respectively. (B) Cultured cardiac fibroblasts were characterized using specific fibroblast markers, vimentin (Vim, green) and discoid in domain receptor-2 (DDR2, red). Cardiomyocytes and endothelial cells were stained using cardiac troponin I (cTnI) and von Willebrand Factor (vWF) to identify the purity of fibroblast isolation. Cellular nuclei were stained using 4', 6-Diamidino-2-Phenylindole (DAPI, blue). Absence of immunofluorescent cTnI and vWF indicated that the cells isolated are cardiac fibroblasts. Scale bar represented $100 \mu \mathrm{m}$.

\subsection{Ang II Enhanced Proliferation and Migration of Cardiac Fibroblasts}

After fibroblasts were originally plated at equal density on day 2, the dose-response effect of Ang II on the proliferative capacity of cardiac fibroblasts was assessed. Compared with non-treated cells (control), stimulation of cardiac fibroblasts with extracellular Ang II for $24 \mathrm{~h}$ significantly increased fibroblast proliferation $(0.9 \pm 0.04$ in $50 \mathrm{nM}$ vs $0.6 \pm 0.07$ in control, $\mathrm{p}<0.05$, Figure $2(\mathrm{~A}))$. The maximal enhancement was seen at a dose range between $50-100 \mathrm{nM}$. Although a higher dose of $300 \mathrm{nM}$ or $1 \mu \mathrm{M}$ also induced fibroblast proliferation, the proliferative degree of fibroblasts started to decline (Figure 2(B)). The calculated values from the control, it was shown that Ang II at different concentrations increased fibroblast proliferation by 1.31, 1.45, 1.43, 1.19 and 1.16 times, respectively. Based on these results, $50 \mathrm{nM}$ of Ang II was selected for subsequent observations.

Transwell migration assay was used to verify the migration of Ang II stimulated cardiac fibroblasts. The cells were seeded in the top compartment of the transwell chamber and the number of migrated cells at the bottom medium through a permeable polycarbonate film was identified. As shown in Figure $2(\mathrm{C})$, the population of purple-stained cells was significantly increased compared 


\section{A}
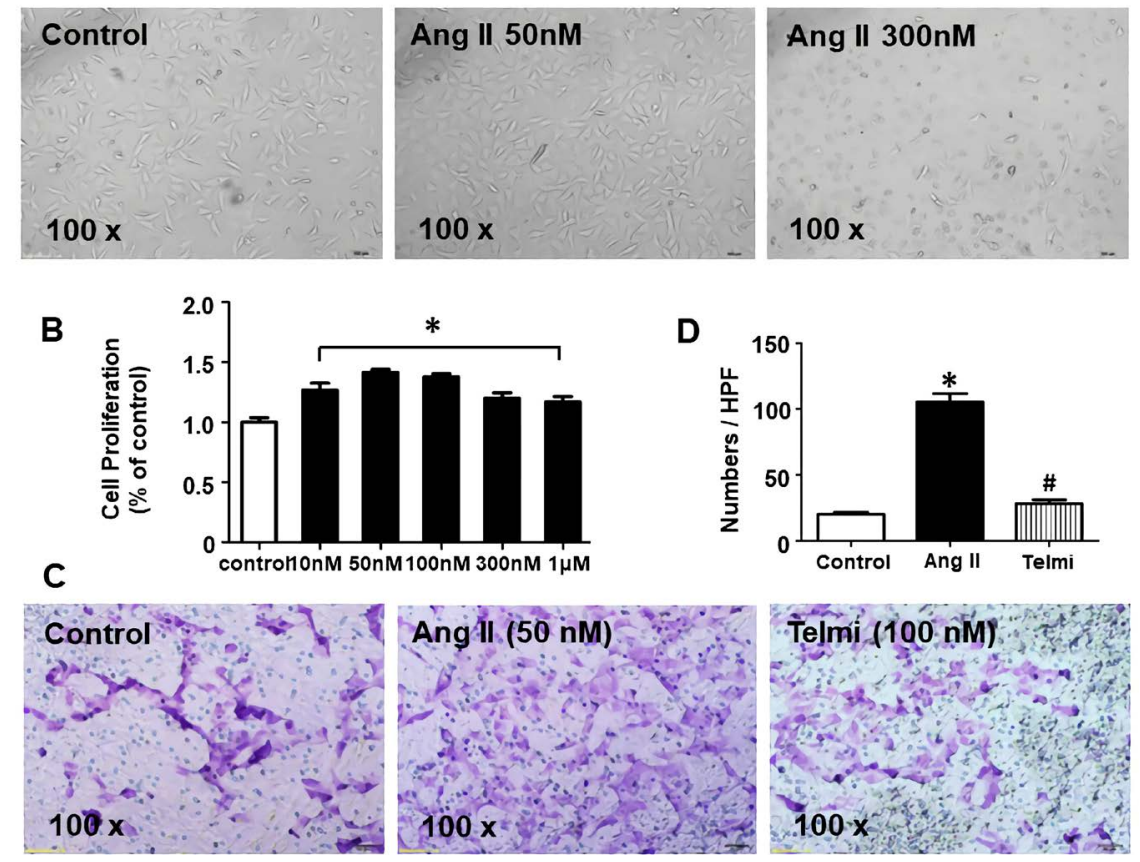

*
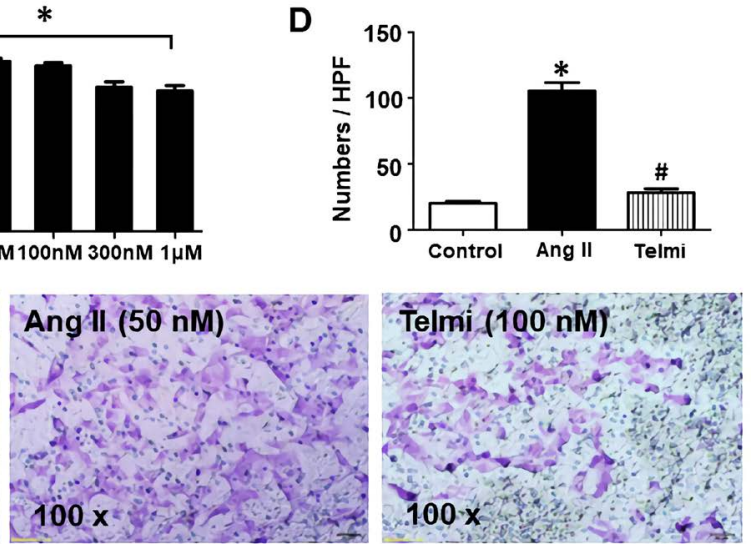

Figure 2. Effect of angiotensin II on proliferation and migration of cardiac fibroblasts. (A) Fibroblast morphology was observed after exposed to concentration of 50 or $300 \mathrm{nM}$ of angiotensin II (Ang II) for $24 \mathrm{~h}$ by a phase-contrast microscopy. (B) Dose response of Ang II on cell viability was evaluated by the CCK-8 assay and the maximal effect was confirmed at $50 \mathrm{nM}$. (C) Cell migration was assessed by Transwell assay after $24 \mathrm{~h}$ of incubation without Ang II (control), Ang II (50 nM) and Ang II plus AT1 receptor blocker, telmisartan (Telmi, $100 \mathrm{nM}$ ). (D) Ang II stimulated cell migration was significantly blocked by Telmi. Values are means \pm SEM of three independent experiments. The cell proliferation without intervention was taken as $100 \%$. Scale bar is $100 \mu \mathrm{m}$.

with the control after treated with Ang II at $50 \mathrm{nM}$. Addition of Ang II AT1 receptor blocker, telmisartan at a dose of $100 \mathrm{nM}$ before Ang II stimulation significantly reduced the number of migrated cells (Figure 2(D)), suggesting an involvement of the AT1 receptor in cell migration.

\subsection{Blockade of Ang II AT1 Receptor Inhibited Expression of TGF- $\beta 1$, Smads and Collagen I}

Stimulation of cardiac fibroblasts by Ang II was accompanied with change in the expression of TGF- $\beta 1$ [9]. Along with enhanced proliferation and migration of cardiac fibroblasts, the protein level of TGF- $\beta 1$ was increased by 1.57 time after $50 \mathrm{nM}$ of Ang II was added (Figure 3(A)), which was significantly blocked by telmisartan, suggesting that the release of TGF- $\beta 1$ from activated fibroblasts is mediated by the AT1 receptor. Consistent with modulation in the production of TGF- $\beta 1$ from fibroblasts by Ang II, the protein levels of pSmad2, Smad4 and collagen I were simultaneously upregulated by Ang II. Compared with the control, the protein levels of pSmad2, Smad4 and collagen I were increased by 1.49, 2.21 and 1.97 times, respectively, which all were blocked by telmisartan treatment (Figures 3(B)-(D)). 


\section{A}
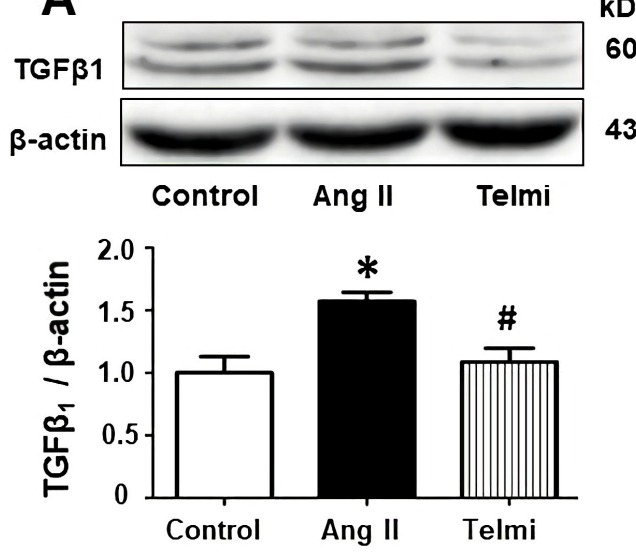

C
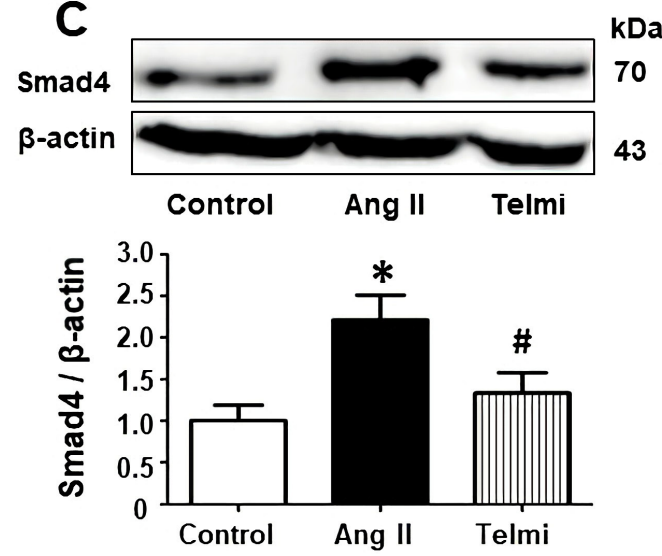

B
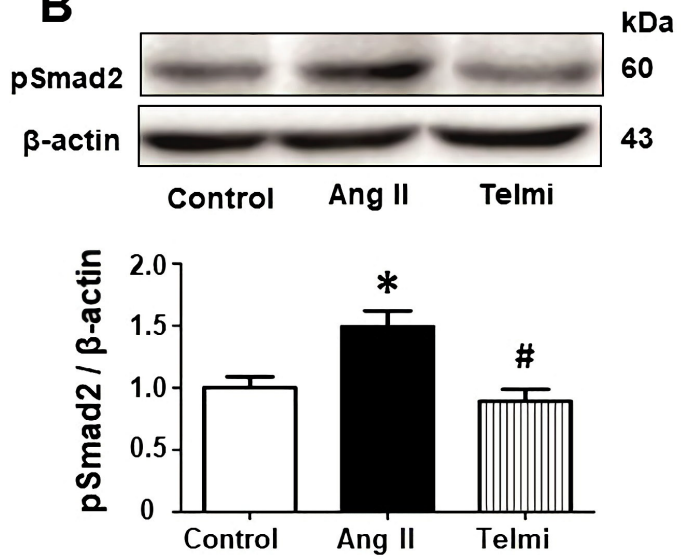

kDa
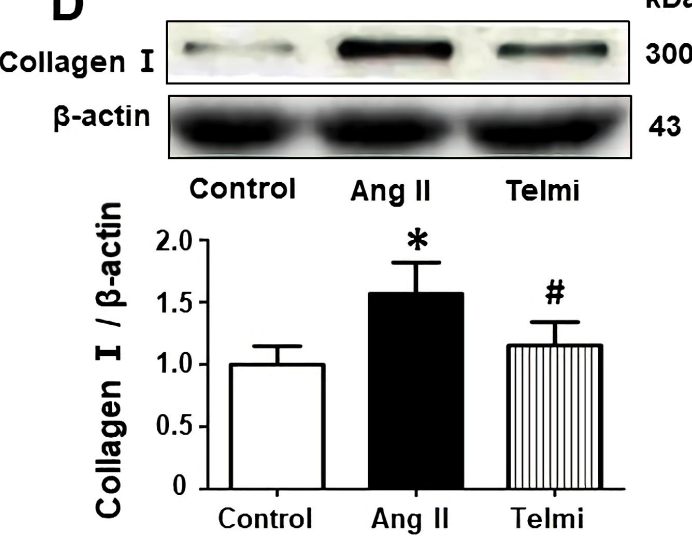

Figure 3. Effect of angiotensin II on the expression of TGF $\beta 1$, pSmad2, Smad4, and collagen I in cardiac fibroblasts. The protein levels were analyzed using Western blot assay in the absence of Ang II (control), Ang II stimulation $(50 \mathrm{nM})$ and Ang II plus AT1 receptor blocker, telmisartan (Telmi, $100 \mathrm{nM}$ ). These protein levels upregulated by Ang II were significantly blocked by Telmi (A)-(D). Results were expressed as a percentage of the control. Data are presented as the means \pm SEM of three independent experiments. ${ }^{*} \mathrm{p}<0.05$ Ang II vs. Control, ${ }^{*} \mathrm{p}<0.05$ Telmi vs. Ang II.

\subsection{Blockade of Ang II AT1 Receptor Downregulated Expression of CD44 mRNA and Protein}

To study whether stimulation of Ang II AT1 receptor is associated with upregulation in the expression of CD44 mRNA and protein, RT-PCR using primers designed to recognize the fibroblast CD44 gene was performed on day 2 to obtain control baseline values for the cell transcriptional activity. Contrary to the expected basal expression of gene transcripts in control, RT-PCR amplified significant detectable amounts of CD44 mRNA in fibroblasts after the cells were exposed to $50 \mathrm{nM}$ of Ang II, when compared to controls (Figure 4(A)). The quantitation of CD44 mRNA expression from a control level revealed that Ang II increases CD44 mRNA by $80.9 \% \pm 4 \%$. Consistent with upregulation of CD44 mRNA, the expression in CD44 protein was also enhanced by $37.9 \% \pm 2 \%$, as shown by Western blot analysis (Figure 4(B)). Addition of the AT1 receptor blocker, telmisartan at a concentration of $100 \mathrm{nM}$ significantly reduced CD44 transcriptional activity $(47.0 \% \pm 3 \%)$ and protein level $(26.2 \% \pm 1 \%)$ relative to 

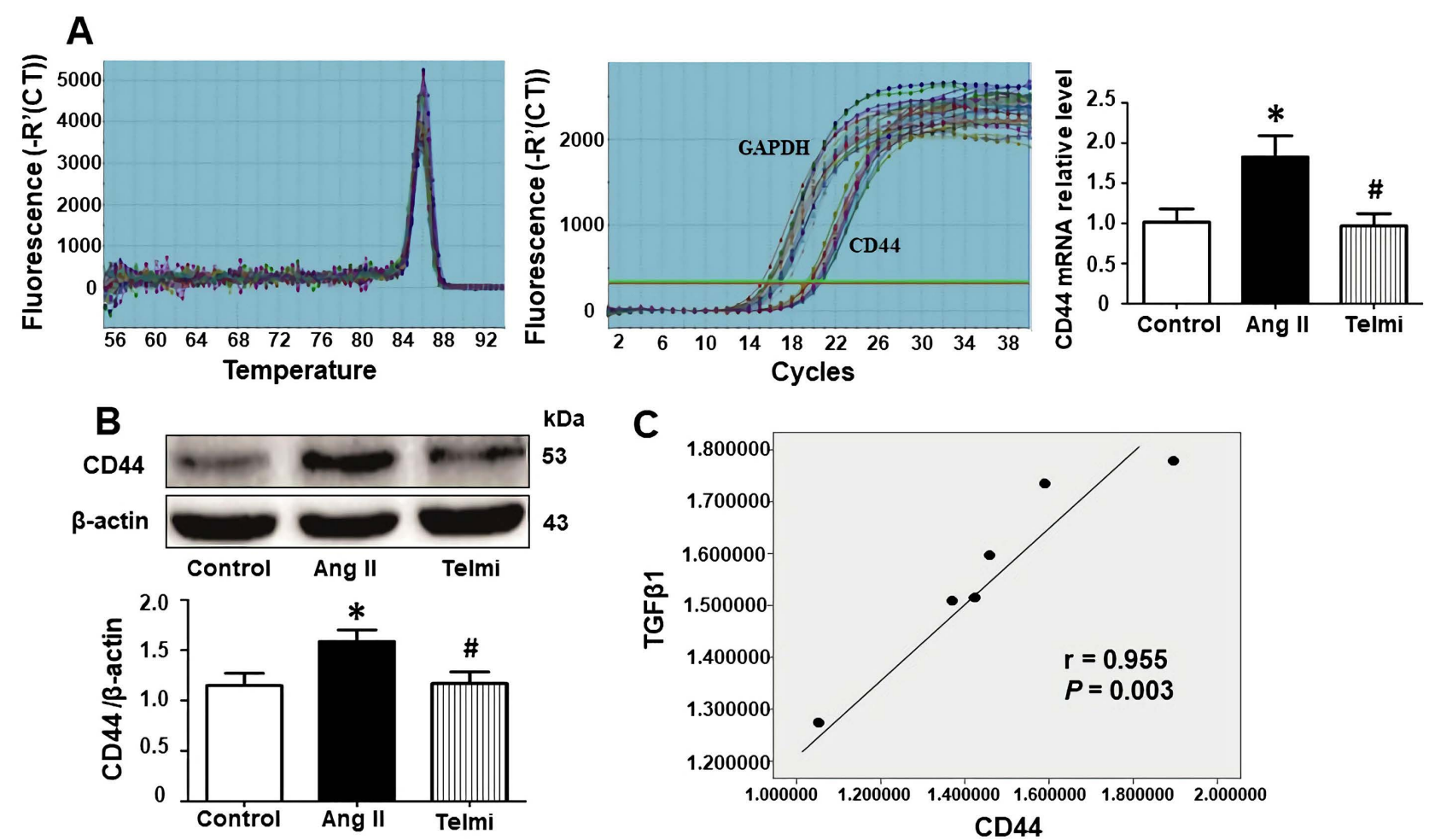

Figure 4. Effects of Ang II on the expression of CD44 in cardiac fibroblasts. The levels of CD44 mRNA and protein were analyzed using RT-PCR and Western blot assay in the absence of Ang II (control), Ang II stimulation (50 nM) and Ang II plus AT1 receptor blocker, telmisartan (Telmi, $100 \mathrm{nM}$ ). Ang II at a dose of $50 \mathrm{nM}$ increased CD44 mRNA (A) and CD44 protein level (B) significantly. Both changes were blocked by Telmi. Pearson correlation test was used as a correlation analysis. There was a positive lineal relationship between TGF $\beta 1$ and CD44 protein (C). Results were expressed as a percentage of the control. Data are presented as the means \pm SEM of three independent experiments. ${ }^{*} \mathrm{p}<0.05$ Ang II vs. Control, ${ }^{*} \mathrm{p}<0.05$ Telmi vs. Ang II.

Ang II stimulation, respectively, (all $\mathrm{p}<0.05$, Figure 4). Furthermore, Pearson correlation analysis showed that there is a linear positive correlation between $\mathrm{CD} 44$ and TGF $\beta 1$, suggesting a role of CD44 in the promotion of TGF $\beta 1$ upregulation (Figure $4(C)$ ).

\subsection{Silencing of CD44 on Cardiac Fibroblasts Abrogated Upregulation of Collagen I Expression}

CD44 was silenced by Ad-CD44 shRNA (siCD44) to further confirm whether CD44 is involved in the activation of cardiac fibroblasts. To validate the effect of siCD44 on the suppression of CD44 in cardiac fibroblasts, RT-PCR and Western blot were carried out. As expected, as negative control, a fluorescent specific shRNA negative (Ad-GFP NC) showed the green fluorescent protein (GFP)-positive cells (Figure 5(A)), which was further confirmed by CD44 mRNA level (Figure $5(B)$ ) and protein expression (Figure $5(C)$ ). Out of the three shRNA constructs, shRNA1 presented the highest efficiency in silencing CD44 expression on cardiac fibroblasts, with a reduction of approximately $29.3 \% \pm 1 \%$, whereas in the other two, Ad-GFP shRNA2 and Ad-GFP shRNA3 did not show significant effects on the number of green fluorescent protein (GFP)-positive cells, when they are compared with the non-silencing control (Ad-GFP NC) (Figure 5(B)). Thus, 

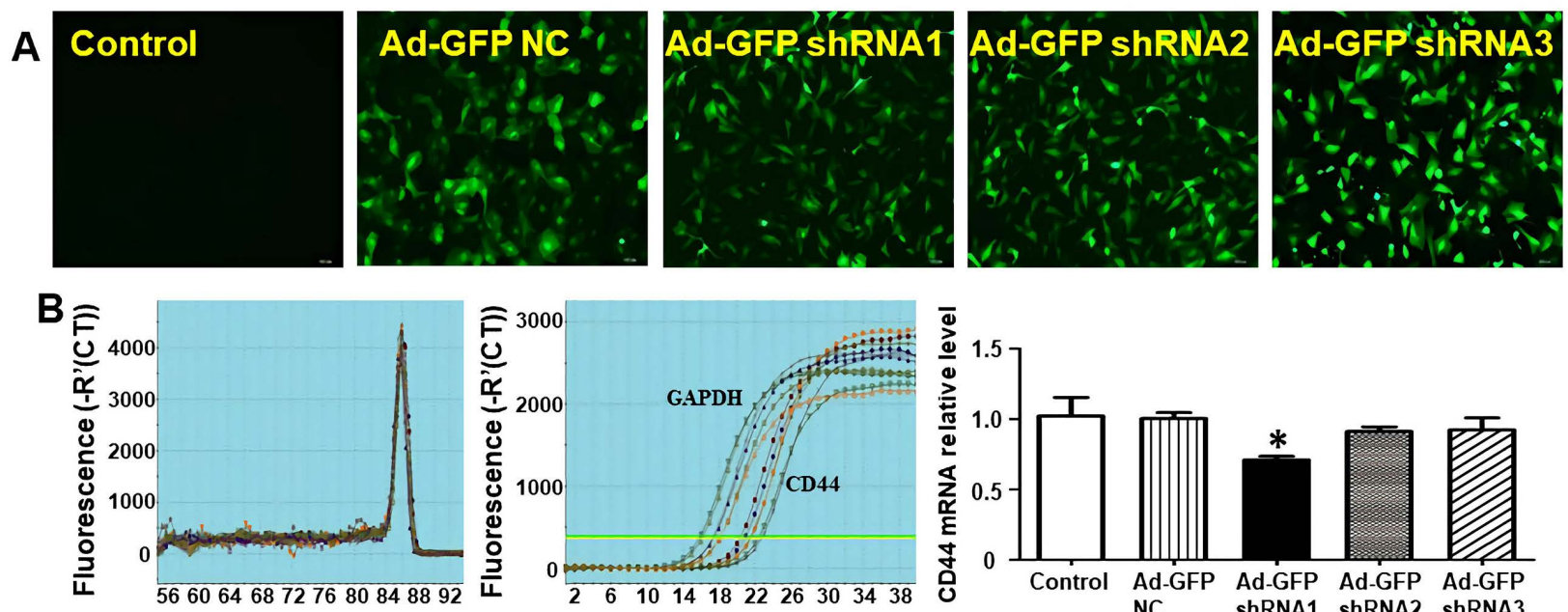

Temperature
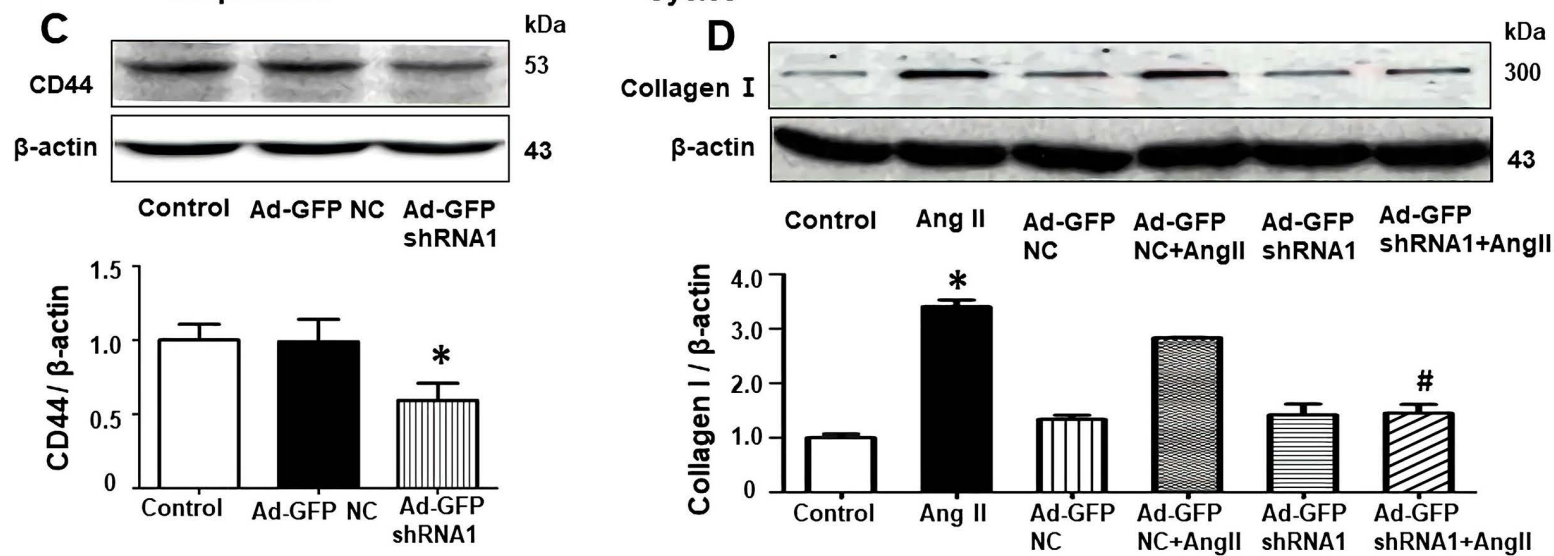

Figure 5. Effect of CD44 silencing on CD44 mRNA, CD44 protein and collagen I in cardiac fibroblasts. The cells were fluorescently labeled by transfecting cardiac fibroblasts with adenovirus not targeting CD44 (Ad-GFP NC) or with adenovirus targeting CD44 (Ad-CD44 shRNA1, Ad-CD44 shRNA2 and Ad-CD44 shRNA3), respectively (A). The reduction in CD44 mRNA (B) and CD44 protein level (C) confirmed an efficacy of CD44 silencing. No increase in the release of collagen I in the presence of Ang II was found when the cells were silenced with Ad-GFP shRNA1 (D). Results are expressed as a percentage of the control level (\% of control). Data are presented as the means \pm SEM of three independent experiments. ${ }^{*} \mathrm{p}<0.05$, Ang II vs. Control, ${ }^{*} \mathrm{p}<0.05$ Ad-GFP shRNA1 plus Ang II vs. Ang II.

Ad-shRNA1 was chosen for sequential establishment of CD44-knockdown on cardiac fibroblasts. Consistent with the results of CD44 silencing, CD44 protein level was also significantly downregulated with Ad-shRNA1 (Figure 5(C)).

Collagen I was selected as a parameter to demonstrate the effect of CD44 knockdown on activation of cardiac fibroblasts. As shown in Figure 5(D), quantification of total collagen I with a computer-assisted densitometric analysis showed a significant increase in collagen I expression after Ang II treatment at $50 \mathrm{nM}$ when compared with control, which was not altered by Ad-GFP NC. However, no significant change in the expression of collagen I was seen when CD44 was silenced with Ad-GFP shRNA1 even after Ang II was re-added, further supporting the role of CD44 in Ang II stimulated cardiac fibroblasts. Based on data demonstrated in this study, we proposed the signaling pathways of Ang II AT1 receptor/CD44 upregulation in mediating the production of collagen (Figure 6). 


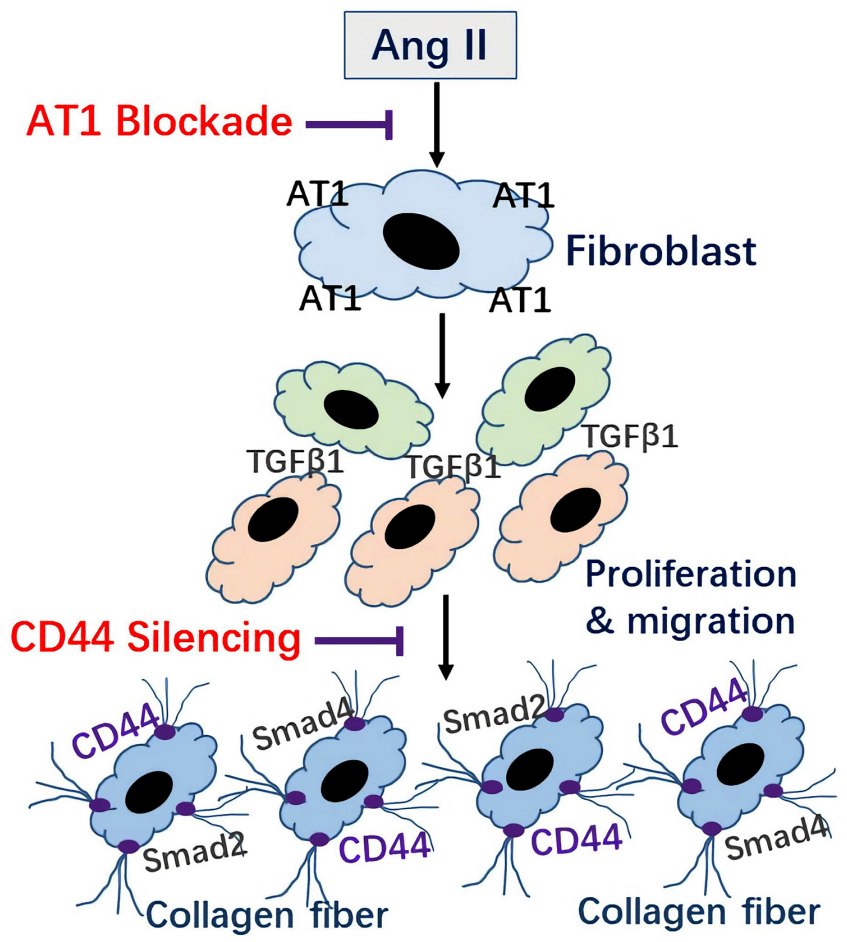

Figure 6. Signaling pathways proposed in the regulation of Ang II AT1 receptor and CD44 receptor mediated collagen production. Direct stimulation of the AT1 receptor increases proliferation and migration of fibroblasts. There is a potential reciprocal interaction between release of TGF $\beta 1$ and activation of CD44 receptor, leading to augmentation in the expression of Smad2/3 and synthesis of collagen from fibroblasts. Cascades underlying Ang II stimulated fibroblasts can be blocked either with the AT1 receptor antagonist or through silencing CD44 receptor.

\section{Discussion}

The goal of this study was to determine whether Ang II has a direct effect on fibroblast proliferation/migration, and how this signaling pathway is transmitted. The key findings of the present study were: 1) the data indicate that Ang II dose-dependently enhances the proliferation/migration of cardiac fibroblasts; 2) the blockade of Ang II AT1 receptor downregulates mRNA and protein levels of CD44, TGF- $\beta 1$, Smad 2 and Smad4, suggesting the AT1 receptor interaction with CD44 in signaling cardiac fibroblast stimulation; 3) silencing of CD44 receptor reduces the synthesis of collagen I, indicating an involvement of CD44 in the activation of cardiac fibroblasts.

Resident cardiac fibroblasts are thought to be predominantly derived from the epithelial cells of the proepicardium undergoing a process termed epithelial-mesenchymal transition during development with approximately $70 \%$ nonmyocytes and 30\% cardiac myocytes, and represent the most abundant nonmyocyte cell population in the heart. Other cell types, including endothelial and vascular smooth muscle cells, make up a small percentage of cell populations in the myocardium [3] [15]. Fibroblasts are distributed throughout the intermyocardium to mediate a variety of cell functions, such as development, differentia- 
tion, migration and morphological changes [16]. In cardiovascular diseases, fibroblasts participate in the development of cardiac remodeling process through cell migration/proliferation and production/secretion of collagens from proliferated fibroblasts, i.e. myofibroblasts [16] [17]. In the in vivo rat model of Ang II infusion, we have previously reported that Ang II elicited fibrotic process is mediated by stimulating the upstream AT1 receptor, primarily located in the blood vessels and myocytes [9]. In the present study, identification of cardiac fibroblasts was based on immunofluorescent staining with vimentin, located in the fibroblast's intermediate filaments, and the collagen receptor Discoidin Domain Receptor 2 (DDR2). DDR2 is the more specific for cardiac fibroblasts because it is not found in cardiac myocytes and endothelial cells. The purity of isolated cardiac fibroblasts was more than $95 \%$ when assayed by this method. Furthermore, these freshly isolated cardiac fibroblasts were not stained by troponin I and Von Willebrand Factor, confirming that cells isolated are not cardiac myocytes and endothelial cells. Data showed clearly that Ang II is capable of stimulating fibroblasts, which is blocked by Ang II AT1 receptor antagonist, telmisartan, providing the direct in vitro evidence to show an involvement of the AT1 receptor in the activation of isolated fibroblasts.

TGF- $\beta 1$ has been shown to be the most influential pro-fibrogenic factor through participating the proliferation, transformation and secretory function of cardiac fibroblasts [18] [19]. In previous in vivo study, we proposed that excessive proliferation/transformation of fibroblasts to myofibroblasts in the extracellular matrix of myocardium is primarily induced by TGF- $\beta 1$, which is released from infiltrated macrophages deriving from the blood circulation [9]. In the present study, in cultured rat heart fibroblasts, TGF- $\beta 1$ expression following Ang II stimulation was upregulated, suggesting that activated fibroblasts also produce TGF- $\beta 1$. In consistent with TGF $\beta-1$ overexpression, Smad2 is phosphorylated and Smad4 expression is upregulated. Binding of Smad2 to the common Smad4 forms Smad complex, which then translocate into the nucleus to regulate the collagen synthesis, as evidenced by upregulated collagen I expression in the present study. In this regard, the conditionally deprived of Smad2 has shown an inhibition of collagen release and reduction of cardiac fibrosis following TGF $\beta-1$ stimulation [20].

The CD44 antigen is a cell-surface glycoprotein and a receptor for glycosaminoglycan hyaluronan (HA), a major component of extracellular matrices. CD44-HA interaction is involved in the production of cardiac fibrosis through cell adhesion, proliferation and migration [7]. In previous in vivo studies, it has been shown that CD44 overexpression plays a critical role in the migration of fibroblasts to sites of injury and differentiation of fibroblasts to myofibroblasts, mainly depending upon Ang II activated TGF- $\beta 1$ signaling pathway [7] [13] [21]. To determine whether CD44 is directly involved in the proliferation of fibroblasts to myofibroblasts, leading to cardiac fibrosis, we selected the in vivo CD44 knockout mouse model to demonstrate that knockout of CD44 expression is associated with the reduction in the upregulation of TGF- $\beta 1$, proliferation of fibroblasts to 
myofibroblasts, synthesis of collagens and formation of cardiac fibrosis [10]. In the present study, we isolated fibroblasts from the neonatal rat heart to determine the relationship between Ang II AT1 receptor and CD44 expression in responsible to fibroblast activation in the absence of HA. We found that Ang II upregulated CD44 expression can be blocked by the AT1 receptor blocker, telmisartan, suggesting that the AT1 receptor is also presented in fibroblasts. Stimulation of the AT1 receptor on fibroblasts may further enhance CD44 expression, and produce TGF- $\beta 1$, as evidenced by a positive linear relationship between CD44 activation and TGF- $\beta 1$ expression. These data indicate that local effect of TGF- $\beta 1$ may depend on the activity of CD44 to control fibroblast proliferation/migration, and to initiate the collagen synthesis. Therefore, CD44 upregulation on fibroblasts may further amplify stimulating effect of Ang II through the AT1 receptor to elicit the cell-cell interactions. To confirm this hypothesis, we determined whether collagen synthesis after Ang II stimulation is directly related with CD44 activation, and silenced CD44 receptor to check the knockout effect on collagen synthesis. Data clearly showed that Ang II promoted collagen secretion is effectively inhibited when CD44 expression is silenced with Ad-GFP shRNA, providing direct evidence showing a role of CD44 in the regulation of collagen synthesis on cardiac fibroblasts. These data were consistent with previous reports showing that $\mathrm{CD} 44$ participates in the activation of TGF- $\beta 1$ and migration of fibroblasts [9] [10].

Study limitations: Angiotensin II stimulates fibroblasts through macrophages-derived TGF- $\beta$ to initiate Smads-mediated collagen production [9]. However, it has been also reported that phosphorylation of Smads depends on p38 mitogen-activated protein kinase (p38MAPK) activity, which can be blocked by p38MAPK inhibitor, suggesting a TGF- $\beta$ stimulation independent pathway [22]. Therefore, more studies are needed to further test whether pharmacologic or siRNA intervention could interfere collagen production without activating TGF- $\beta$ in a cellular level. Moreover, stimulation of the AT1 receptor by angiotensin II is associated with fibroblast activation [9]. It is unknown whether pharmacologic blockade of the AT1 receptor or knockout of the AT1 receptor could modulate p38MAPK signaling, and reduce collagen production. Foundational studies in cultured fibroblasts are necessary to demonstrate potential efficacy of the AT1 receptor blocker relative to the p38MAPK inhibitor in attenuation of collagen production.

\section{Conclusion}

In summary, upregulation of the AT1 receptor and over expression of CD44 occur on cardiac fibroblasts when these cells are stimulated by Ang II, leading to the recruitment of multiple signaling pathways in the production of collagen. Either blockade of the AT1 receptor or silencing of CD44 expression reduces TGF- $\beta 1 /$ Smads-mediated collagen synthesis. These data provide cellular and molecular evidence that, in addition to Ang II induced cardiac fibrosis through stimulating multiple cell types including endothelial cells, macrophages and car- 
diomyocytes, Ang II also directly stimulates fibroblasts. Therefore, therapeutic advance to attenuate Ang II induced myocardial injury could be achieved by specifically targeting anti-fibroblast activation [23].

\section{Acknowledgements}

This study was supported by the PhD Foundation Program of Shanxi Medical University (BS0320627).

\section{Conflicts of Interest}

The authors state that there is no conflict of interest.

\section{References}

[1] Camelliti, P., Borg, T.K. and Kohl, P. (2005) Structural and Functional Characterization of Cardiac Fibroblasts. Cardiovascular Research, 65, 40-51. https://doi.org/10.1016/j.cardiores.2004.08.020

[2] Goldsmith, E.C., Hoffman, A., Morales, M.O., Potts, J.D., Price, R.L., McFadden, A., Rice, M. and Borg, T.K. (2004) Organization of Fibroblasts in the Heart. Developmental Dynamics, 230, 787-794. https://doi.org/10.1002/dvdy.20095

[3] Banerjee, I., Fuseler, J.W., Price, R.L., Borg, T.K. and Baudino, T.A. (2007) Determination of Cell Types and Numbers During Cardiac Development in the Neonatal and Adult Rat and Mouse. American Physiological Society, 293, H1883-H1891. https://doi.org/10.1152/ajpheart.00514.2007

[4] Krenning, G., Zeisberg, E.M. and Kalluri, R. (2010) The Origin of Fibroblasts and Mechanism of Cardiac Fibrosis. Journal of Cellular Physiology, 225, 631-637. https://doi.org/10.1002/jcp.22322

[5] Frangogiannis, N.G. (2019) Cardiac Fibrosis: Cell Biological Mechanisms, Molecular Pathways and Therapeutic Opportunities. Molecular Aspects of Medicine, 65, 70-99. https://doi.org/10.1016/j.mam.2018.07.001

[6] Chistiakov, D.A., Orekhov, A.N. and Bobryshev, Y.V. (2016) The Role of Cardiac Fibroblasts in Post-Myocardial Heart Tissue Repair. Experimental and Molecular Pathology, 101, 231-240. https://doi.org/10.1016/j.yexmp.2016.09.002

[7] Suleiman, M., Abdulrahman, N., Yalcin, H. and Mraiche, F. (2018) The Role of CD44, Hyaluronan and NHE1 in Cardiac Remodeling. Life Sciences, 209, 197-201. https://doi.org/10.1016/j.lfs.2018.08.009

[8] Midgley, A.C., Rogers, M., Hallett, M.B., Clayton, A., Bowen, T., Phillips, A.O. and Steadman, R. (2013) Transforming Growth Factor- $\beta 1$ (TGF- $\beta 1$ )-Stimulated Fibroblast to Myofibroblast Differentiation Is Mediated by Hyaluronan (HA)-Facilitated Epidermal Growth Factor Receptor (EGFR) and CD44 Co-Localization in Lipid Rafts. The Journal of Biological Chemistry, 288, 14824-1438. https://doi.org/10.1074/jbc.M113.451336

[9] Bai, F., Pang, X.F., Zhang, L.H., Wang, N.P., McKallip, R.J., Garner, R.E. and Zhao, Z.Q. (2016) Angiotensin II AT1 Receptor Alters ACE2 Activity, eNOS Expression and CD44-Hyaluronan Interaction in Rats with Hypertension and Myocardial Fibrosis. Life Sciences, 153, 141-152. https://doi.org/10.1016/j.lfs.2016.04.013

[10] Yang, L.W., Qin, D.Z., James, E, McKallip, R.J., Wang, N.P., Zhang, W.W., Zheng, R.H., Han, Q.H. and Zhao, Z.Q. (2019) CD44 Deficiency in Mice Protects the Heart against Angiotensin II-Induced Cardiac Fibrosis. Shock, 51, 372-380. 
https://doi.org/10.1097/SHK.0000000000001132

[11] Pellegrin, M., Bouzourène, K., Aubert, J.F., Nahimana, A., Duchosal, M.A. and Mazzolai, L. (2018) Activation of Bone Marrow-Derived Cells Angiotensin (Ang) II Type 1 Receptor by Ang II Promotes Atherosclerotic Plaque Vulnerability. International Journal of Molecular Sciences, 19, E2621. https://doi.org/10.3390/ijms19092621

[12] Singhania, G., Ejaz, A.A., McCullough, P.A., Kluger, A.Y., Balamuthusamy, S., Dass, B., Singhania, N. and Agarwal, A. (2019) Continuation of Chronic Heart Failure Therapies During Heart Failure Hospitalization: A Review. Reviews in Cardiovascular Medicine, 20, 111-120. https://doi.org/10.31083/j.rcm.2019.03.562

[13] Tzircotis, G., Thorne, R.F. and Isacke, C.M. (2005) Chemotaxis towards Hyaluronan Is Dependent on CD44 Expression and Modulated by Cell Type Variation in CD44-Hyaluronan Binding. Journal of Cell Science, 118, 5119-5128.

https://doi.org/10.1242/jcs.02629

[14] Siddesha, J.M., Valente, A.J., Sakamuri, S.S., Yoshida, T., Gardner, J.D., Somanna, N., Takahashi, C., Noda, M. and Chandrasekar, B. (2013) Angiotensin II Stimulates Cardiac Fibroblast Migration via the Differential Regulation of Matrixins and RECK. Journal of Molecular and Cellular Cardiology, 65, 9-18. https://doi.org/10.1016/j.yjmcc.2013.09.015

[15] Bertagnolli, M., Dios, A., Béland-Bonenfant, S., Gascon, G., Sutherland, M., Lukaszewski, M.A., Cloutier, A., Paradis, P., Schiffrin, E.L. and Nuyt, A.M. (2016) Activation of the Cardiac Renin-Angiotensin System in High Oxygen-Exposed Newborn Rats: Angiotensin Receptor Blockade Prevents the Developmental Programming of Cardiac Dysfunction. Hypertension, 67, 774-782. https://doi.org/10.1161/HYPERTENSIONAHA.115.06745

[16] Ivey, M.J. and Tallquist, M.D. (2016) Defining the Cardiac Fibroblast. Circulation Journal, 80, 2269-2276. https://doi.org/10.1253/circj.CJ-16-1003

[17] Porter, K.E. and Turner, N.A. (2009) Cardiac Fibroblasts: At the Heart of Myocardial Remodeling. Pharmacology \& Therapeutics, 123, 255-278. https://doi.org/10.1016/j.pharmthera.2009.05.002

[18] Weiskirchen, R., Weiskirchen, S. and Tacke, F. (2019) Organ and Tissue Fibrosis: Molecular Signals, Cellular Mechanisms and Translational Implications. Molecular Aspects of Medicine, 65, 2-15. https://doi.org/10.1016/j.mam.2018.06.003

[19] Chen, M.M., Lam, A., Abraham, J.A., Schreiner, G.F. and Joly, A.H. (2000) CTGF Expression Is Induced by TGF-Beta in Cardiac Fibroblasts and Cardiac Myocytes: A Potential Role in Heart Fibrosis. Journal of Molecular and Cellular Cardiology, 32, 1805-1819. https://doi.org/10.1006/jmcc.2000.1215

[20] Zent, J. and Guo, L.W. (2018) Signaling Mechanisms of Myofibroblastic Activation: Outside-in and Inside-out. Cellular Physiology and Biochemistry, 49, 848-868. https://doi.org/10.1159/000493217

[21] Khalil, H., Kanisicak, O., Prasad, V., Correll, RN., Fu, X., Schips, T., Vagnozzi, R.J., Liu, R., Huynh, T., Lee, S.J., Karch, J. and Molkentin, J.D. (2017) Fibroblast-Specific TGF- $\beta$-Smad2/3 Signaling Underlies Cardiacfibrosis. Journal of Clinical Investigation, 127, 3770-3783. https://doi.org/10.1172/JCI94753

[22] Rodríguez-Vita, J., Sánchez-López, E., Esteban, V., Rupérez, M., Egido, J. and Ruiz-Ortega, M. (2005) Angiotensin II Activates the Smad Pathway in Vascular Smooth Muscle Cells by a Transforming Growth Factor-Beta-Independent Mechanism. Circulation, 111, 2509-2517. https://doi.org/10.1161/01.CIR.0000165133.84978.E2 
[23] Gourdie, R.G., Dimmeler, S. and Kohl, P. (2016) Novel Therapeutic Strategies Targeting Fibroblasts and Fibrosis in Heart Disease. Nature Reviews Drug Discovery, 15, 620-638. https://doi.org/10.1038/nrd.2016.89 